\title{
Delivery of Exogenous Antigens to Induce Cytotoxic CD8+ T Lymphocyte Responses
}

\author{
Julia Kim, Vandana Gambhir, Attiya Alatery, and Sameh Basta \\ Department of Microbiology and Immunology, Queen's University, Kingston, ON, Canada K7L 3N6 \\ Correspondence should be addressed to Sameh Basta, bastas@queensu.ca
}

Received 11 December 2009; Accepted 9 March 2010

Academic Editor: Kim Klonowski

Copyright () 2010 Julia Kim et al. This is an open access article distributed under the Creative Commons Attribution License, which permits unrestricted use, distribution, and reproduction in any medium, provided the original work is properly cited.

Vaccines intended to induce a cytotoxic CD8+ T-cell response are highly sought after. However, some of these vaccines can be problematic if they replicate in the host. An alternative strategy is to exploit cross-presentation of exogenous antigens to express peptides on major histocompatibility complex (MHC) class I molecules. During cross-presentation, the delivered exogenous antigen can be taken up and processed through diverse mechanisms. Here, we will discuss the recent advances regarding the complex nature of the cross-priming process and the models that reflect its relevance in vivo. Moreover, we summarize current data that explore potential adjuvants and vaccine vectors that deliver antigens to activate CD8+ T cells relying on cross-presentation.

\section{Introduction}

Naïve CD8+ T cells become activated when their receptors recognize antigens presented by professional antigenpresenting cells (pAPCs) in the context of MHC-I molecules. Upon recognition of target cells, such primed cytotoxic Tlymphocytes (CTLs) are able to limit the spread of virus infection through the lysis of host-infected cells. Moreover, along with helper $\mathrm{T}$ cells they orchestrate the induction of key cytokines such as interferon- $\gamma$ (IFN- $\gamma$ ) and tumor necrosis factor- $\alpha$ (TNF- $\alpha$ ) needed for an optimal immune response [1]. In addition to their importance in halting virus replication, CTLs play a central role in the specific immune response and are essential in the elimination of intracellular pathogens and limiting the potential escape of tumor cells $[2,3]$.

The cross-presentation pathway allows for exogenouslyderived antigens to be presented on MHC-I molecules to CTLs [4-7]. To induce antitumor immune responses, or to prime CTLs for viruses that inhibit direct presentation, the immune system utilizes cross-presentation $[8,9]$. Thus, cross-presentation represents a promising mechanism for strategies that target the induction of CTL responses for vaccine development to induce both effector and protective memory T-cell responses. In order for us to discuss the role of cross-presentation in future vaccine developments we need to explore antigen presentation in details.

\section{The MHC-I Presentation Pathways}

All nucleated cells express MHC-I molecules and are capable of presenting antigens to CTLs. However, the "priming" stage or activation of naïve $\mathrm{CD} 8+\mathrm{T}$ cells requires peptide-MHC class I complexes presented by the pAPC in addition to costimulatory signals such as interaction with $\mathrm{B} 7$ molecules, CD40, CD70, and the 4-1BBL family members [10], as well as the secretion of key cytokines such as IL-12 and IFN- $\alpha$ [1113]. These cytokines are also important in optimal CD8+ Tcells memory development [14]. The CD8 $+\mathrm{T}$ cells priming step can occur via two different mechanisms of antigen presentation: the direct- and cross-presentation pathways. In the direct or "endogenous" presentation pathway, antigens are derived from endogenously synthesized proteins, improperly translated proteins, and, or unstable defective ribosomal proteins $[15,16]$. These cytosolic proteins are targeted for proteasomal degradation after their polyubiquination. Proteasomal degradation products are then transported into the endoplasmic reticulum (ER) via the transporter associated with antigen processing (TAP), moving through the Golgi complex to the cell surface (Figure 1).

Alternatively, the cross- or the "exogenous-" presentation pathway occurs when uninfected pAPCs present exogenously-derived antigens $[4,5,7]$ after uptake of soluble or cell-associated antigens. The latter antigenic form can 


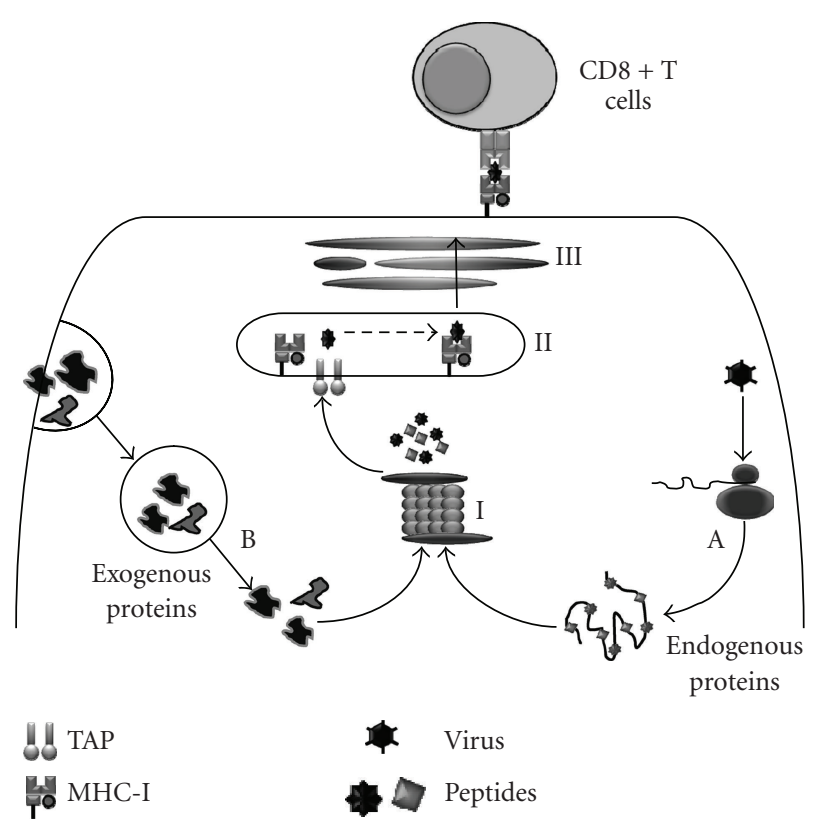

FIGURE 1: Antigen processing pathways. Direct presentation involves the processing of endogenously synthesized antigens (route A) by the proteasome (I) to break them down into smaller fragment (polypeptides) that are transported through the transporter associated with antigen processing (TAP) into the endoplasmic reticulum (II) for loading onto newly synthesized MHC class I molecules. These peptide-loaded MHC class I molecules are then transported through the Golgi (III) to the cell surface for presentation to $\mathrm{CD} 8+\mathrm{T}$ cells. Considering the major route for antigen crosspresentation (route B), exogenous antigens are internalized in the endosome/phagosome before they are released into the cytosol and degraded by the proteasome to be presented on MHC class I molecules.

be derived from different sources such as cellular fragments [17-19], intracellular bacteria [20], virus-infected or tumor cells [21-26], and as reported recently from parasitic infections [27]. It appears that protein stability in these models is critical for efficient cross-presentation to occur $[17,28-31]$. On the other hand, soluble proteins tend to be cross-presented, but with much lower efficiency than cellassociated proteins [32]. Although efficient presentation of exogenous antigens was originally attributed to macrophages [33], it is now clear that such function can be achieved by different bone marrow-derived APCs, including DCs and macrophages [34-36], as well as spleen-derived macrophages [37]. Interestingly, it appears both spleen and bone marrowderived macrophages down regulate their ability to crosspresent cell-associated antigens during differentiation [37].

In cross-presentation, several processing mechanisms have been proposed [38], including the canonical model where antigens inside the endosomal/phagosomal vesicles are translocated into the cytosol before they follow the regular proteasome/ER/TAP route (Figure 1). In addition, it was proposed that pAPCs possess organelles, such as the phagosome, optimized for the cross-presentation of exogenous antigens [38], by functioning autonomously to generate MHC class I-peptide complexes from proteins internalized into the same phagosome. Moreover, soluble antigen cross-presentation was recently found to involve early endocytic compartment trafficking that is aided by TAP recruitment and signaling through TLR4 and MyD88 [39]. However, whether these antigen processing models are applicable to exogenous antigens other than OVA proteins has yet to be investigated.

\section{Induction of Tolerance versus Priming}

As this paper focuses on exogenous antigen delivery in vaccine development, we will concentrate on cross-presentation. Regardless of the exact processing mechanism in play within the cell, the cross-presentation pathway can result in one of two outcomes: cross-tolerance or cross-priming. Inadequate activation of CTLs may result in tolerance, which is an ideal situation for self-antigens. Thus, under normal circumstances, cross-presentation of peripheral self-antigens from normal healthy tissue will induce cross-tolerance [ 40 , 41]. This outcome could depend on many factors such as which APC is presenting the antigen [41-43], and how the antigen is being cross-presented $[44,45]$. On the other hand, if immunity is to be induced, signals necessary for T-cell activation [46] leading to cross-priming will be provided by pAPCs, leading to clonal expansion, differentiation, and establishment of robust memory cells.

Memory $\mathrm{T}$ cells are long-lived cells that allow for an efficient adaptive immune response upon re-exposure to a pathogen [47]. Such memory cells can be divided into two categories depending on their trafficking and effector functions. The effector memory $\mathrm{T}$ cells $\left(\mathrm{T}_{\mathrm{EM}}\right)$ are found in peripheral tissues, can respond rapidly to infections, and are characterized by the low expression of certain markers such as CD62L, CCR7, and CD27. Upon antigen recognition, they immediately produce effector cytokines, such as IFN- $\gamma$ and TNF- $\alpha$, conferring a state of protection [47]. In contrast, central memory $\mathrm{T}$ cells $\left(\mathrm{T}_{\mathrm{CM}}\right)$, which are found mainly in secondary lymphatic organs such as lymph nodes, express high levels of the CD26L, CCR7, and CD27 markers, and are slow to exhibit their effector functions. An important goal of any vaccination protocol would be the induction of both sets of CD8+ T-cell memory [48].

Another critical factor to consider in T-cell activation focuses on the profile of T-cell immunodominance hierarchy that a vaccine is likely to induce. With regard to immunodominance, epitope-specific CD8+ T cells can be organized into a hierarchy, in which certain immunodominant epitopes will cause a set of $\mathrm{T}$ cells to expand extensively compared to subdominant epitope-specific T cells [49]. A CTL response could be more effective when generated against a greater number of epitopes, as it is more diverse and should lead to the formation of a wider number of specific memory CD8+ T-cell populations that can confer protective immunity.

In evaluating the physiological relevance of crosspriming in vivo to vaccine development, it has been demonstrated that cross-priming is a robust process that induces significant CTL responses to multiple epitopes, both 
in viral and tumor models [21]. In a related study, crosspriming of inactivated flu virus resulted in a broad and balanced CTL responses compared to live virus. Interestingly, it appears that the ability of an epitope to access cross-priming may support its immunodominance position when considering the overall hierarchy [50-52]. During virus challenge experiments, it was found that an initial cross-priming with a lymphocytic choriomeningitis virus (LCMV)-nucleoprotein (NP), that normally cross-primes CTLs for the NP396 epitope [17], resulted in increasing the magnitude of NP396 epitope-specific T cells. Thus, the immunodominance hierarchy was modified so that the NP396 epitope, an immunodominant epitope found within the LCMV-NP, was favored in a subsequent virus challenge [50]. These effects were maintained over time and may have important implications for vaccination protocols such as the currently administrated inactivated flu vaccines. In this paper, it was speculated that the initial cross-priming with LCMV-NP resulted in the enhanced ability of NP396-specific clones, but not the NP205-specifc clones, to expand and out compete other T-cell clones specific for epitopes generated from other proteins [50]. Importantly, these changes only occurred when cross-priming preceded viral infection and thus when T-cell frequencies competing for resources were lower. Such findings were not observed in the vaccinia virus model when peptides were used prior to infections to prime CTL responses [53]. These diverse findings may be related to the different replication profiles of both viruses considering that vaccinia does not replicate as efficiently as LCMV in mice. It would also be interesting to compare immunodominance data in a virus challenge experiment obtained after priming with peptide versus proteins, where in the latter condition cross-priming is expected to take place $[50,53]$.

Finally, with regard to cross-priming and vaccination, the discovery and development of adjuvants that are able to regulate cross-presentation is of utmost importance. When co-administered with an antigen, adjuvants assist in the generation of an immune response. Adjuvants can elicit their immune enhancing effects in a variety of ways, including targeting pattern recognition receptors such as toll-like receptors (TLRs) [54]. TLR signaling can result in DC maturation, leading to upregulated $\mathrm{MHC}$ and co-stimulatory molecule expression, and increased priming of $\mathrm{T}$ cells without the need for CD4 T-cell help [26, 54-57]. For example, it has been shown that the synthetic TLR9 ligand, CpG-ODN (Cytosinephosphorothioate-guanine oligodeoxynucleotides), is readily taken up by receptor-mediated endocytosis and was found to enhance CTL responses. Furthermore, the antigen-specific primary and secondary expansion of $\mathrm{T}$ cells was better established when TLR9 ligand was cross-linked to the OVA antigen $[55,58-60]$.

Thus, based on many models of cross-priming, researchers are logically aiming to develop novel vaccine vectors that are capable of inducing CTL responses in vivo by utilizing exogenous antigen delivery. In the latter part of this paper, we will discuss some examples of such vectors and contemplate on other future developments in the vaccine field.

\section{Utilizing Cross-Presentation in the Induction of CTL Responses}

In vaccination, delivering exogenous antigens via crosspresentation should ideally induce both humoral and CTL responses to improve effective immunity. As the major pAPC in vivo, DCs are able to stimulate naïve $\mathrm{CD} 8+\mathrm{T}$ cells and are important when considering precise targeting of delivery vectors. A key area of research involves the modification of vector surfaces with ligands for DC receptors, such as TLR ligands, as well the application of natural substances that can allow for effective immune modulation. For example, the active metabolites of vitamin D3 can influence adaptive immune responses to peripherally administered antigens during vaccination/infection. In these experiments, the ability of murine DCs to migrate from skin sites of vaccination to mucosal lymphoid organs seem to be dependent on the production of active vitamin D3 metabolites produced locally at the vaccination site, which was associated with the application of specific TLR (TLR3/TLR4) ligands [61, $62]$. These findings support previous studies, which demonstrated that both systemic and common mucosal immune responses developed in adult mice upon administration of a subcutaneous or intradermal vaccine containing active vitamin D3 [63]. Thus, vitamin D3 and its metabolites may represent potential novel adjuvant preparations.

Generally, vaccines should induce effective protective CTL immunity, and the production of immunological memory. Potent CTL responses have been reported to be limited to live attenuated viral or bacterial vaccines [48]. However, the use of such vaccines is offset by the risk of reinitiating virulence. Thus, a key target of novel vectors, such as virus-like particles, microparticles, and archaeosomes, is to efficiently access the cross-presentation pathway. As a result, these vectors will induce CTL effector and memory T-cell responses without risking the health of the host. Such vectors have shown interesting immunological properties, discussed below, that enable them to be potent in inducing crosspriming.

4.1. Virus-Like Particles. Virus-like particles (VLPs) are formed from viral structural proteins that lack a viral genome and thus assemble into nonreplicative particles [64, 65]. VLPs are safe, stable, and extremely immunogenic due to their highly repetitive molecular structures $[64,65]$. In addition to their highly immunogenic nature, VLPs can be easily modified to increase their immunogenicity by packaging CpGs into the core antigen particle, which results in higher frequencies of peptide-specific CD8+ $\mathrm{T}$ cells being induced [66].

Since VLPs are exogenously acquired by pAPCs, they are able to use the cross-presentation pathway [67-69] and can induce both CTL and memory responses [70]. For example, a recombinant parvovirus- (PPV- ) VLP, encoding a known LCMV CD8+ T-cell epitope, was able to stimulate the immune system up to 8 months after the last immunization [65]. This long-lasting effect was characterized by protection against a lethal viral (LCMV) infection, in addition to 
responses against peptide-coated or virus-infected target cells [65]. The high particulate nature of PPV-VLPs was hypothesized to be associated with its optimal delivery to the MHC-class I antigen presentation pathway, resulting in high immunogenicity [65]. This ability to induce CTLs via cross-priming was demonstrated in another study with HIV p55gag-VLPs immunizations [70]. In this study, immunized animals were efficiently primed ( $>8.5$ months) for p55gagspecific CTL responses that recognized multiple HIV p55gag epitopes. Altogether, these studies demonstrate that VLPs are able to prime strong MHC-I restricted CD8+ T-cell responses through cross-presentation.

The exact mechanism of cross-presentation is not fully defined; however, recent data suggests that the processing pathway involving VLPs may vary according to the type of vector employed. For example, cross-presentation of papaya mosaic VLPs, which induces robust CTL responses [71], was proteasome independent [67]. In the case of parvovirus-like particles (PPV-VLPs) without adjuvant, exogenous antigen was localized in the late endosomes of DCs [72]. It is important to note that presence of potential adjuvants, such as TLR ligands [39], enhances the efficiency of crosspresentation as it allows for the relocation of the TAP molecules to the early endosomes. As one would expect, the processing of the PPV-VLPs required vacuolar acidification, proteasome activity, and TAP translocation, but not MHC class I molecule recycling. Altogether, the data shows that the cross-presentation of PPV-VLPs occurs via an endosometo-cytosol processing pathway [72]. Interestingly, the uptake and capture of PPV-VLPs involved macropinocytosis and lipid rafts participation.

In contrast when employing hepatitis B virus (HBV) VLPs, it was found that efficient CTL cross-priming by DCs occurred either in a TAP-dependent or TAP-independent manner [69]. The HBV (VLPs) are also taken up by macropinocytosis, but rely on endosomal processing and recycling MHC I molecules in DCs [73].

Thus it may be necessary to evaluate each VLP type independently, as each vector will have a unique nature that may be closely associated with the protection it can elicit against its targeted pathogen or disease. It is also important to note that not all VLPs are able to activate pAPCs by themselves. This was highlighted by data showing that a CTL epitope from LCMV (p33-VLPs) was efficiently processed for MHC class I presentation but induced weak CTL responses [66]. The CTL response failed to mediate effective protection from viral challenge in the absence of external substances that activate APCs, such as anti-CD40 antibodies or $\mathrm{CpG}$ oligonucleotides, which engage TLR9 [66]. Clearly, further research is required to fully optimize this technology, however, VLPs represent a promising avenue for vector development. Recently, a novel approach utilized HIV-1 VLPs of a mutant HIV-1 Nef, which acts as an anchoring element for foreign proteins. Immunization with such VLPs incorporating a HPV-16 E7 protein, fused to the mutant HIV-1 Nef, resulted in an robust anti-E7 CD8+ T-cell response, and protection against an HPV-E7 expressing tumour [74]. Furthermore, it was suggested that co-inoculation of VLPs with diverse epitopes of the same pathogen could be used to generate CTLs with a wider range of specificities to attain enhanced protection [74].

4.2. Microspheres (MSs). Microspheres (MSs) are composed of biodegradable polymers, such as poly (lactic acid) (PLA), poly(glycolic acid) (PGA), and polylactic-co-glycolic acid (PLGA) [75], and can be formed through processes such as solvent evaporation or spray drying $[54,75]$. Depending on their diameter, loaded MSs can be taken up by APCs through phagocytosis [76]. This uptake could be also enhanced due to the depot formation at the site of injection [54]. MSs can be administered orally because they can protect their cargo including adjuvants (e.g., CpG-ODNs) from enzyme-mediated degradation [77]. Upon hydrolysis of their polymeric bonds by phagosomal enzymes after phagocytosis, the MS polymers are disrupted, causing the release of the encapsulated antigen [54].

Biodegradable microspheres (MSs) are useful to microencapsulate antigens alone or combined with adjuvants. Their ability to allow for the controlled release of the antigen or to allow for the formation of a depot at the site of injection is particularly useful [78]. Poly ( $\mathrm{D}$, L-lactide-co-glycolide) (PLGA) has been used to prepare microspheres that possess tremendous potential to release encapsulated antigens in a controlled manner, thus facilitating the protection of the antigen cargo from immediate degradation in vivo [79]. A key advantage of MSs is the flexibility in their design, which allows for a variety of possibilities in combining different antigens and adjuvants. Moreover, specific cell targeting is feasible through the addition of ligands to the vehicle surface that are able to specifically bind receptors on the surface of the targeted cell type $[78,79]$.

MSs can deliver exogenous antigens to the crosspresentation pathway, but appear to require additional signals, such as adjuvants, to trigger a CTL response [80]. This process may involve T-cell help which can be provided via CD40L interactions on helper $\mathrm{T}$ cells, and is needed to promote the costimulatory activation state of DCs required for optimal cross-priming $[81,82]$. As MSs are unable to trigger DC maturation by themselves [83], cross-presentation of antigens by immature DCs can result in T-cell ignorance [43].

Data examining this phenomenon concur that additional help signals are required to enhance the cross-priming of MSs. For example, MSs encapsulating an antigen with an immunodominant CD8+ T-cell epitope (B-OVAp) failed to induce antigen-specific cross-priming of CD8+ T cells, unless recombinant PPV-VLP were co-injected, which served as an adjuvant to induce a potent CTL response [80]. Interestingly, the PPV-VLPs enhanced this CTL response in $\mathrm{MHC}$ class $\mathrm{II}^{-/-}$and $\mathrm{CD} 40^{-/-}$mice indicating that the CD4+ T-cell help was bypassed under these conditions. Furthermore, such adjuvant activity did not require TLR2, TLR4, or TLR9 stimulation.

In accordance, recent research has focused on examining the influence of adjuvants on cross-priming within the antigen-containing MSs. These studies used OVA incorporated into MSs and examined cross-primed antigen-specific 
T cells by the secretion of IFN- $\gamma$ in peptide specific assays $[60,84]$. The CTL response elicited when TLR ligands were coencapsulated with OVA was stronger compared with OVA-MS alone or if antigen and adjuvants were separately encapsulated.

In a study examining the coencapsulation of OVA and a TLR9 ligand (CpG-ODN), the authors found that both OVA and CpG-ODN were translocated into lysosomal-associated membrane protein-1 (Lamp1)-positive phago-endosomal compartments of DC [60]. The processed OVA peptides on MHC class I and II molecules on DCs allowed for robust antigen-specific $\mathrm{CD} 4+$ and $\mathrm{CD} 8+\mathrm{T}$-cell proliferation that were TLR9-dependent [60]. The processing route for MHCI antigen presentation in this study required endosomal acidification, TAP translocation, and proteasomal processing [60]. In a separate study, it was found that CpG-ODN in the MSs induced DC maturation, characterized by increased MHC and co-stimulatory molecule expression, which may have aided the increased cross-priming [84]. In both reports $[60,84]$, induction of OVA specific Tcells, especially after boosting, was comparable to what is observed when live vectors encoding OVA are employed $[85,86]$. In addition, the induced immunity was protective against a challenge of L. monocytogenes, vaccinia virus and protected against OVA expressing tumors $[60,84]$.

However, including adjuvant activity with the antigens is not limited solely to MSs. For example, liposomes are vesicles composed of lipid bilayers that are separated by aqueous regions, and are comparable to MSs as they are also biodegradable and safe, making them attractive vectors for vaccine delivery $[75,87]$. Liposomes were one of the early vectors developed in order to co-deliver adjuvants, such as CpG ODN, and antigens [87], and have been used in therapy of diseases such as leishmaniasis [88]. In addition, the adjuvant effects of liposomes complexed to TLR agonists were found to induce effective CD4+ and CD8+ T-cell responses against peptide and protein antigens. In particular TLR3 or TLR9 agonists effectively cross-primed CD8+ T-cell responses independently of CD4+ T-cell help [56].

Nonetheless, despite the advantages of using MSs in vaccine delivery, additional data explaining the exact processing mechanisms is needed. In addition, further research is required to reveal how this generates protective potential when applying different antigenic determinants in different infections models. Regardless, the simultaneous inclusion of antigen and adjuvant appears to hold promise to optimize future vaccination strategies that use MSs.

4.3. Archaeosomes. Archaeosomes are a novel generation of liposomes that are composed of the polar lipids of Archaea, which leads to immune-stimulating interactions with APCs. The archeal lipid cores offer archaeosomes longlasting stability at a variety of environmental extremes, including temperature, $\mathrm{pH}$, and oxidative conditions. These properties allow them to induce strong memory responses and avoid fusion or aggregation, thus reducing antigen leakage [89]. The entrapment of antigen in archaeosomes has been documented to facilitate MHC-I cross-presentation, resulting in the activation of long-lived CD8 + T-cell immunity [85, 89-91]. For example, a single immunization with a Methanobrevibacter smithii-OVA (M. smithii) archaeosome was found to prime $2-5 \%$ of antigen-specific $\mathrm{T}$ cells by day 7 , while boosting on day 21 resulted in an expansion to about $20 \%$ [85]. Furthermore, after two immunizations of low doses M. smithii-OVA archaeosomes, re-stimulated spleen cell effectors were able to exhibit strong cytolytic activity of target cells labeled with the specific peptides [92]. This ability to activate CTLs with archaeosomes was demonstrated in $\mathrm{CD} 4^{-/-}[93]$ and IL-12 $2^{-/-}$mice [92].

When examining the quality of archaeosome-primed CD8+ T-cell responses, it was reported that the responses were superior in magnitude to other particulate vesicular systems such as liposomes [90]. In addition, a single injection of the vector induced a profound primary response, leading to the formation of around $1 \%$ of $\mathrm{CD} 8+\mathrm{T}_{\mathrm{CM}}$ which exhibited a phenotype $\left(\mathrm{CD} 44^{++} \mathrm{CD} 62 \mathrm{~L}^{++}\right)$typical of the central memory cells [85]. The responses were comparable to vaccination with live vectors encoding OVA such as $L$. monocytogenes vector [85] and induced protective CD8+ Tcells responses that were TLR2-independent.

Unlike the MSs delivery system that we discussed above, archaeosomes can target antigen for cross-presentation while simultaneously activating pAPC, thus allowing for proper induction of co-stimulatory molecules and cytokine production [89]. OVA entrapped in M. smithii is the bestcharacterized model with respect to studying the mechanisms of archaeosome cross-presentation. Due to the presence of exposed archaetidylserine head groups on their surfaces, M. smithii archaeosomes are taken up by receptormediated endocytosis [91]. Thus, they are able to utilize the same pathway that relies on the phosphatidylserine (PS) receptor-mediated clearance of apoptotic cells by pAPCs [91]. However, after phagocytosis, it was seen that the presentation of archaeosomal-antigens on MHC-I relied on cytosolic proteasomal processing and TAP-activity. Interestingly, it was demonstrated that the blocking of endosomal acidification resulted in the block of MHC I processing, which can be due to the fact that acidification in the endosomes or phagolysosomes helps antigen escape from the archaeosomes [91].

It is important to note that other archaeosome types lacking PS are also phagocytosed efficiently and can induce strong CD8+ T-cell immunity [89]. Thus, different archaeosome vectors can be expected to rely on different receptors for cellular entry. Overall, archaeosomes provide promise to the field of vaccine development, and appear to represent versatile, potentially universal vectors. This research field can only benefit from the involvement and collaboration of more research groups.

\section{Conclusion}

The majority of vaccines utilized in the past were attenuated vaccine strains and, although critical in the prevention of several infectious diseases, the inherent risk that they carry necessitates the development of alternative vaccines. 
TABLE 1: Summary of the diverse vector approaches used for the generation of CTLs.

\begin{tabular}{|c|c|c|}
\hline Vector & Outcome & Ref \\
\hline \multirow{2}{*}{$\begin{array}{l}\text { VLP - Recombinant porcine } \\
\text { parvovirus encoding LCMV-NP }\end{array}$} & $\begin{array}{l}\text { - Strong CTL responses without a need for adjuvant, the CTL activity persisted in vivo } \\
\text { for } 8 \text { months }\end{array}$ & \multirow[t]{2}{*}[65]{} \\
\hline & - Complete protection against lethal LCMV infection & \\
\hline VLP - HIV-p55gag-VLP & $\begin{array}{l}\text { - Without adjuvant, long lived CTL responses against multiple HIV-1 p55gag epitopes } \\
\text { were detected }\end{array}$ & {$[70]$} \\
\hline $\begin{array}{l}\text { VLP - Papaya mosaic VLP } \\
\text { (LCMV-GP33) }\end{array}$ & $\begin{array}{l}\text { - Immunized mice develop GP33-specific CTLs, which rapidly expanded post-LCMV } \\
\text { challenge and enhanced the protection against LCMV infection in dose-dependent } \\
\text { manner }\end{array}$ & {$[67]$} \\
\hline VLP - HBV-VLP (LCMV-GP33) & $\begin{array}{l}\text { - TAP-deficient DC and macrophages mediated cross-presentation of GP33 in vivo and } \\
\text { in vitro }\end{array}$ & [69] \\
\hline $\begin{array}{l}\text { VLP- VSV encoding HPV-16 } \\
\text { E7protein }\end{array}$ & $\begin{array}{l}\text { - anti-E7 CD8+ T-cell response which conferred protection against E7 expressing tumor } \\
\text { cells }\end{array}$ & {$[74]$} \\
\hline Microsphere - Co-injected & - Results in the priming of potent CTL responses in CD4 and CD40-independent manner & [80] \\
\hline & - Protective CTL against the OVA-bearing melanoma & \\
\hline \multirow[t]{4}{*}{ Microsphere - PLGA-MSs } & $\begin{array}{l}\text { - Vaccination enhanced CTL responses when OVA was coencapsulated with CpG or } \\
\text { polyI:C }\end{array}$ & \multirow[t]{2}{*}{84} \\
\hline & $\begin{array}{l}\text { - Single immunization with coencapsulated MS-OVA-CpG induced efficient CTLs and } \\
\text { protected against infection with OVA-expressing vaccinia virus }\end{array}$ & \\
\hline & - Clonal expansion of primary and secondary antigen-specific CD4 and CD8 T cells & \multirow{2}{*}{60} \\
\hline & - Potency demonstrated by protective immune responses to either infection or tumors & \\
\hline \multirow{3}{*}{$\begin{array}{l}\text { Liposomes -liposome-Ag-nucleic } \\
\text { acid complexes (LANAC) }\end{array}$} & $\begin{array}{l}\text { - TLR3 or TLR9 were able to enhance CTL cross-priming independent of CD4+ T-cell } \\
\text { help }\end{array}$ & \multirow[t]{3}{*}{56} \\
\hline & $\begin{array}{l}\text { - Antigen-specific CD8+ T cells were functionally active and persisted for long periods in } \\
\text { tissues }\end{array}$ & \\
\hline & - Effective immunity against B16 tumors and M. tuberculosis & \\
\hline \multirow{8}{*}{$\begin{array}{l}\text { Archaeosome (M. smithii)- } \\
\text { Encoding OVA }\end{array}$} & - Single injection evoked profound primary CTL response & \multirow{3}{*}{85} \\
\hline & - Recall response observed $>300$ days & \\
\hline & - Protective CD8+ T cells induced in TLR2-deficient mice & \\
\hline & - Resisted tumor growth of B16OVA melanoma cells & \multirow{2}{*}{92} \\
\hline & - Enhanced CTL responses in the absence of IL-12 and IFN- $\gamma$ & \\
\hline & - CTL activity was undetectable in perforin-deficient mice & \multirow{3}{*}{93} \\
\hline & - Long-term responses in CD4+ $\mathrm{T}$ cell deficient mice & \\
\hline & - Potent memory CTL response to OVA lasting for $\geq 154$ days & \\
\hline
\end{tabular}

The advent of nonreplicating vaccine vectors (key findings summarized in Table 1) has revealed the importance of efficiently targeting exogenously derived antigens to immune cells, which would allow for the cross-priming of CD8+ $\mathrm{T}$ cells rather than cross-tolerance. The attractiveness of this mechanism is offset by the need to optimize such vectors, in order to maximize the immune response. In order to achieve this goal, more research is needed to dissect the cellular and molecular factors that regulate crosspresentation events during the initiation of CTL immune responses. We have come far since the observations made by Edward Jenner and the field is now ripe for developing novel vaccine-adjuvant complexes to make the next step forward that improves on the initial discovery of the small pox vaccine.

\section{Acknowledgment}

This work is supported by grants from the Dairy Farmers of Canada and Natural Sciences and Engineering Research Council of Canada.

\section{References}

[1] S. Shresta, C. T. Pham, D. A. Thomas, T. A. Graubert, and T. J. Ley, "How do cytotoxic lymphocytes kill their targets?" Current Opinion in Immunology, vol. 10, no. 5, pp. 581-587, 1998.

[2] C. J. M. Melief and W. M. Kast, "Cytotoxic T lymphocyte therapy of cancer and tumor escape mechanisms," Seminars in Cancer Biology, vol. 2, no. 5, pp. 347-354, 1991. 
[3] J. T. Harty, R. D. Schreiber, and M. J. Bevan, "CD8 T cells can protect against an intracellular bacterium in an interferon $\gamma$ independent fashion," Proceedings of the National Academy of Sciences of the United States of America, vol. 89, no. 23, pp. 11612-11616, 1992.

[4] S. Basta and A. Alatery, "The cross-priming pathway: a portrait of an intricate immune system," Scandinavian Journal of Immunology, vol. 65, no. 4, pp. 311-319, 2007.

[5] W. R. Heath, G. T. Belz, G. M. N. Behrens, et al., "Crosspresentation, dendritic cell subsets, and the generation of immunity to cellular antigens," Immunological Reviews, vol. 199, pp. 9-26, 2004.

[6] J. W. Yewdell and S. M. M. Haeryfar, "Understanding presentation of viral antigens to $\mathrm{CD}^{+} \mathrm{T}$ cells in vivo: the key to rational vaccine design," Annual Review of Immunology, vol. 23, pp. 651-682, 2005.

[7] L. Shen and K. L. Rock, "Priming of T cells by exogenous antigen cross-presented on MHC class I molecules," Current Opinion in Immunology, vol. 18, no. 1, pp. 85-91, 2006.

[8] S. Basta and J. R. Bennink, "A survival game of hide and seek: cytomegaloviruses and MHC class I antigen presentation pathways," Viral Immunology, vol. 16, no. 3, pp. 231-242, 2003.

[9] R. G. van der Most, A. Currie, B. W. S. Robinson, and R. A. Lake, "Cranking the immunologic engine with chemotherapy: using context to drive tumor antigen cross-presentation towards useful antitumor immunity," Cancer Research, vol. 66, no. 2, pp. 601-604, 2006.

[10] A. H. Sharpe, "Mechanisms of costimulation," Immunological Reviews, vol. 229, no. 1, pp. 5-11, 2009.

[11] J. Valenzuela, C. Schmidt, and M. F. Mescher, "The roles of IL-12 in providing a third signal for clonal expansion of naive CD8 T cells," The Journal of Immunology, vol. 169, no. 12, pp. 6842-6849, 2002.

[12] J. M. Curtsinger, J. O. Valenzuela, P. Agarwal, D. Lins, and M. F. Mescher, "Cutting edge: type I IFNs provide a third signal to CD8 T cells to stimulate clonal expansion and differentiation," The Journal of Immunology, vol. 174, no. 8, pp. 4465-4469, 2005.

[13] M. F. Mescher, P. Agarwal, K. A. Casey, C. D. Hammerbeck, Z. Xiao, and J. M. Curtsinger, "Molecular basis for checkpoints in the CD8 T cell response: tolerance versus activation," Seminars in Immunology, vol. 19, no. 3, pp. 153-161, 2007.

[14] Z. Xiao, K. A. Casey, S. C. Jameson, J. M. Curtsinger, and M. F. Mescher, "Programming for CD8 T cell memory development requires IL-120 or type I IFN," The Journal of Immunology, vol. 182, no. 5, pp. 2786-2794, 2009.

[15] K. L. Rock, I. A. York, T. Saric, and A. L. Goldberg, "Protein degradation and the generation of MHC class I-presented peptides," Advances in Immunology, vol. 80, pp. 1-70, 2002.

[16] J. W. Yewdell, E. Reits, and J. Neefjes, "Making sense of mass destruction: quantitating MHC class I antigen presentation," Nature Reviews Immunology, vol. 3, no. 12, pp. 952-961, 2003.

[17] S. Basta, R. Stoessel, M. Basler, M. van den Broek, and M. Groettrup, "Cross-presentation of the long-lived lymphocytic choriomeningitis virus nucleoprotein does not require neosynthesis and is enhanced via heat shock proteins," The Journal of Immunology, vol. 175, no. 2, pp. 796-805, 2005.

[18] J. F. Fonteneau, D. G. Kavanagh, M. Lirvall, et al., "Characterization of the MHC class I cross-presentation pathway for cell-associated antigens by human dendritic cells," Blood, vol. 102, no. 13, pp. 4448-4455, 2003.
[19] F. R. Carbone and M. J. Bevan, "Class I-restricted processing and presentation of exogenous cell-associated antigen in vivo," Journal of Experimental Medicine, vol. 171, no. 2, pp. 377-387, 1990.

[20] J. D. Pfeifer, M. J. Wick, R. L. Roberts, K. Findlay, S. J. Normark, and C. V. Harding, "Phagocytic processing of bacterial antigens for class I MHC presentation to T cells," Nature, vol. 361, no. 6410, pp. 359-362, 1993.

[21] W. Chen, K.-A. Masterman, S. Basta, et al., "Cross-priming of $\mathrm{CD}^{+} \mathrm{T}$ cells by viral and tumor antigens is a robust phenomenon," European Journal of Immunology, vol. 34, no. 1, pp. 194-199, 2004.

[22] K. Shimizu, Y. Kurosawa, M. Taniguchi, R. M. Steinman, and S.-I. Fujii, "Cross-presentation of glycolipid from tumor cells loaded with $\alpha$-galactosylceramide leads to potent and longlived T cell-mediated immunity via dendritic cells," Journal of Experimental Medicine, vol. 204, no. 11, pp. 2641-2653, 2007.

[23] Y. Li, L.-X. Wang, P. Pang, et al., "Cross-presentation of tumor associated antigens through tumor-derived autophagosomes," Autophagy, vol. 5, no. 4, pp. 576-577, 2009.

[24] S. Basta, W. Chen, J. R. Bennink, and J. W. Yewdell, "Inhibitory effects of cytomegalovirus proteins US2 and US11 point to contributions from direct priming and cross-priming in induction of vaccinia virus-specific $\mathrm{CD}^{+} \mathrm{T}$ cells," The Journal of Immunology, vol. 168, no. 11, pp. 5403-5408, 2002.

[25] A. Serna, M. C. Ramirez, A. Soukhanova, and L. J. Sigal, "Cutting edge: efficient MHC class I cross-presentation during early vaccinia infection requires the transfer of proteasomal intermediates between antigen donor and presenting cells," The Journal of Immunology, vol. 171, no. 11, pp. 5668-5672, 2003.

[26] O. Schulz, S. S. Diebold, M. Chen, et al., "Toll-like receptor 3 promotes cross-priming to virus-infected cells," Nature, vol. 433, no. 7028, pp. 887-892, 2005.

[27] R. S. Goldszmid, I. Coppens, A. Lev, P. Caspar, I. Mellman, and A. Sher, "Host ER-parasitophorous vacuole interaction provides a route of entry for antigen cross-presentation in Toxoplasma gondii-infected dendritic cells," Journal of Experimental Medicine, vol. 206, no. 2, pp. 399-410, 2009.

[28] C. C. Norbury, S. Basta, K. B. Donohue, et al., "CD8 ${ }^{+}$T cell cross-priming via transfer of proteasome substrates," Science, vol. 304, no. 5675, pp. 1318-1321, 2004.

[29] L. Shen and K. L. Rock, "Cellular protein is the source of crosspriming antigen in vivo," Proceedings of the National Academy of Sciences of the United States of America, vol. 101, no. 9, pp. 3035-3040, 2004.

[30] A. D. Bins, M. C. Wolkers, M. D. van den Boom, J. B. A. G. Haanen, and T. N. M. Schumacher, "In vivo antigen stability affects DNA vaccine immunogenicity," The Journal of Immunology, vol. 179, no. 4, pp. 2126-2133, 2007.

[31] M. C. Wolkers, N. Brouwenstijn, A. H. Bakker, M. Toebes, and T. N. M. Schumacher, "Antigen bias in T cell cross-priming," Science, vol. 304, no. 5675, pp. 1314-1317, 2004.

[32] M. Li, G. M. Davey, R. M. Sutherland, et al., "Cell-associated ovalbumin is cross-presented much more efficiently than soluble ovalbumin in vivo," The Journal of Immunology, vol. 166, no. 10, pp. 6099-6103, 2001.

[33] M. Kovacsovics-Bankowski, K. Clark, B. Benacerraf, and K. L. Rock, "Efficient major histocompatibility complex class I presentation of exogenous antigen upon phagocytosis by macrophages," Proceedings of the National Academy of Sciences of the United States of America, vol. 90, no. 11, pp. 4942-4946, 1993. 
[34] L. J. Sigal and K. L. Rock, "Bone marrow-derived antigenpresenting cells are required for the generation of cytotoxic $\mathrm{T}$ lymphocyte responses to viruses and use transporter associated with antigen presentation (TAP)-dependent and -independent pathways of antigen presentation," Journal of Experimental Medicine, vol. 192, no. 8, pp. 1143-1150, 2000.

[35] L. J. Sigal, S. Crotty, R. Andino, and K. L. Rock, "Cytotoxic T-cell immunity to virus-infected non-haematopoietic cells requires presentation of exogenous antigen," Nature, vol. 398, no. 6722, pp. 77-80, 1999.

[36] A. Y. Huang, P. Golumbek, M. Ahmadzadeh, E. Jaffee, D. Pardoll, and H. Levitsky, "Bone marrow-derived cells present MHC class I-restricted tumour antigens in priming of antitumour immune responses," Ciba Foundation Symposium, vol. 187, pp. 229-244, 1994.

[37] A. Alatery, S. Siddiqui, M. Chan, A. Kus, E. O. Petrof, and S. Basta, "Cross, but not direct, presentation of cell-associated virus antigens by spleen macrophages is influenced by their differentiation state," Immunology and Cell Biology, vol. 88, no. 1, pp. 3-12, 2010.

[38] A. L. Ackerman and P. Cresswell, "Cellular mechanisms governing cross-presentation of exogenous antigens," Nature Immunology, vol. 5, no. 7, pp. 678-684, 2004.

[39] S. Burgdorf, C. Scholz, A. Kautz, R. Tampe, and C. Kurts, "Spatial and mechanistic separation of cross-presentation and endogenous antigen presentation," Nature Immunology, vol. 9, no. 5, pp. 558-566, 2008.

[40] C. Kurts, H. Kosaka, F. R. Carbone, J. F. A. P. Miller, and W. R. Heath, "Class I-restricted cross-presentation of exogenous self-antigens leads to deletion of autoreactive CD8 ${ }^{+}$T cells," Journal of Experimental Medicine, vol. 186, no. 2, pp. 239-245, 1997.

[41] A. Limmer, J. Ohl, C. Kurts, et al., "Efficient presentation of exogenous antigen by liver endothelial cells to $\mathrm{CD}^{+} \mathrm{T}$ cells results in antigen-specific T-cell tolerance," Nature Medicine, vol. 6, no. 12, pp. 1348-1354, 2000.

[42] A. Schildknecht, H. C. Probst, K. D. McCoy, et al., "Antigens expressed by myelinating glia cells induce peripheral crosstolerance of endogenous CD8 ${ }^{+} \mathrm{T}$ cells," European Journal of Immunology, vol. 39, no. 6, pp. 1505-1515, 2009.

[43] M. B. Lutz and C. Kurts, "Induction of peripheral CD4 $4^{+}$T-cell tolerance and CD8 ${ }^{+}$T-cell cross-tolerance by dendritic cells," European Journal of Immunology, vol. 39, no. 9, pp. 2325-2330, 2009.

[44] W. L. Redmond, C.-H. Wei, H. T. C. Kreuwel, and L. A. Sherman, "The apoptotic pathway contributing to the deletion of naive CD8 $\mathrm{T}$ cells during the induction of peripheral tolerance to a cross-presented self-antigen," The Journal of Immunology, vol. 180, no. 8, pp. 5275-5282, 2008.

[45] A. Limmer, J. Ohl, G. Wingender, et al., "Cross-presentation of oral antigens by liver sinusoidal endothelial cells leads to CD8 T cell tolerance," European Journal of Immunology, vol. 35, no. 10, pp. 2970-2981, 2005.

[46] I. Mellman and R. M. Steinman, "Dendritic cells: specialized and regulated antigen processing machines," Cell, vol. 106, no. 3, pp. 255-258, 2001.

[47] J. K. Northrop and H. Shen, "CD8 ${ }^{+}$T-cell memory: only the good ones last," Current Opinion in Immunology, vol. 16, no. 4, pp. 451-455, 2004.

[48] M. T. Esser, R. D. Marchese, L. S. Kierstead, et al., "Memory T cells and vaccines," Vaccine, vol. 21, no. 5-6, pp. 419-430, 2003.

[49] J. W. Yewdell, "Confronting complexity: real-world immunodominance in antiviral $\mathrm{CD}^{+} \mathrm{T}$ cell responses," Immunity, vol. 25, no. 4, pp. 533-543, 2006.
[50] E. Dunbar, A. Alatery, and S. Basta, "Cross-priming of a single viral protein from lymphocytic choriomeningitis virus alters immunodominance hierarchies of $\mathrm{CD}^{+} \mathrm{T}$ cells during subsequent viral infections," Viral Immunology, vol. 20, no. 4, pp. 585-598, 2007.

[51] V. Pavelic, M. Matter, S. Mumprecht, I. Breyer, and A. F. Ochsenbein, "CTL induction by cross-priming is restricted to immunodominant epitopes," European Journal of Immunology, vol. 39, no. 3, pp. 704-716, 2009.

[52] P. Otahal, S. C. Hutchinson, L. M. Mylin, M. J. Tevethia, S. S. Tevethia, and T. D. Schell, "Inefficient cross-presentation limits the $\mathrm{CD}^{+} \mathrm{T}$ cell response to a subdominant tumor antigen epitope," The Journal of Immunology, vol. 175, no. 2, pp. 700-712, 2005.

[53] Y. Wang, I. E. A. Flesch, and D. C. Tscharke, "Vaccinia virus $\mathrm{CD}^{+} \mathrm{T}$-cell dominance hierarchies cannot be altered by prior immunization with individual peptides," Journal of Virology, vol. 83, no. 17, pp. 9008-9012, 2009.

[54] A. Heit, D. H. Busch, H. Wagner, and F. Schmitz, "Vaccine protocols for enhanced immunogenicity of exogenous antigens," International Journal of Medical Microbiology, vol. 298, no. 1-2, pp. 27-32, 2008.

[55] A. Heit, F. Schmitz, M. O’Keeffe, et al., "Protective CD8 T cell immunity triggered by CpG-protein conjugates competes with the efficacy of live vaccines," The Journal of Immunology, vol. 174, no. 7, pp. 4373-4380, 2005.

[56] K. Zaks, M. Jordan, A. Guth, et al., "Efficient immunization and cross-priming by vaccine adjuvants containing TLR3 or TLR9 agonists complexed to cationic liposomes," The Journal of Immunology, vol. 176, no. 12, pp. 7335-7345, 2006.

[57] S. Hervas-Stubbs, A. Olivier, F. Boisgerault, N. Thieblemont, and C. Leclerc, "TLR3 ligand stimulates fully functional memory $\mathrm{CD}^{+} \mathrm{T}$ cells in the absence of $\mathrm{CD} 4^{+}$T-cell help," Blood, vol. 109, no. 12, pp. 5318-5326, 2007.

[58] A. Heit, T. Maurer, H. Hochrein, et al., "Cutting edge: Tolllike receptor 9 expression is not required for CpG DNA-aided cross-presentation of DNA-conjugated antigens but essential for cross-priming of CD8 T cells," The Journal of Immunology, vol. 170, no. 6, pp. 2802-2805, 2003.

[59] A. Heit, K. M. Huster, F. Schmitz, M. Schiemann, D. H. Busch, and H. Wagner, "CpG-DNA aided cross-priming by crosspresenting B cells," The Journal of Immunology, vol. 172, no. 3, pp. 1501-1507, 2004.

[60] A. Heit, F. Schmitz, T. Haas, D. H. Busch, and H. Wagner, "Antigen co-encapsulated with adjuvants efficiently drive protective T cell immunity," European Journal of Immunology, vol. 37, no. 8, pp. 2063-2074, 2007.

[61] E. Y. Enioutina, D. Bareyan, and R. A. Daynes, "TLR-induced local metabolism of vitamin D3 plays an important role in the diversification of adaptive immune responses," The Journal of Immunology, vol. 182, no. 7, pp. 4296-4305, 2009.

[62] E. Y. Enioutina, D. Bareyan, and R. A. Daynes, "TLR ligands that stimulate the metabolism of vitamin D3 in activated murine dendritic cells can function as effective mucosal adjuvants to subcutaneously administered vaccines," Vaccine, vol. 26, no. 5, pp. 601-613, 2008.

[63] R. A. Daynes, E. Y. Enioutina, S. Butler, H.-H. Mu, Z. A. McGee, and B. A. Araneo, "Induction of common mucosal immunity by hormonally immunomodulated peripheral immunization," Infection and Immunity, vol. 64, no. 4, pp. 1100-1109, 1996.

[64] E. V. L. Grgacic and D. A. Anderson, "Virus-like particles: passport to immune recognition," Methods, vol. 40, no. 1, pp. 60-65, 2006. 
[65] C. Sedlik, M.-F. Saron, J. Sarraseca, I. Casal, and C. Leclerc, "Recombinant parvovirus-like particles as an antigen carrier: a novel nonreplicative exogenous antigen to elicit protective antiviral cytotoxic T cells," Proceedings of the National Academy of Sciences of the United States of America, vol. 94, no. 14, pp. 7503-7508, 1997.

[66] T. Storni, C. Ruedl, K. Schwarz, R. A. Schwendener, W. A. Renner, and M. F. Bachmann, "Nonmethylated CG motifs packaged into virus-like particles induce protective cytotoxic T cell responses in the absence of systemic side effects," The Journal of Immunology, vol. 172, no. 3, pp. 1777-1785, 2004.

[67] D. Leclerc, D. Beauseigle, J. Denis, et al., "Proteasomeindependent major histocompatibility complex class I crosspresentation mediated by papaya mosaic virus-like particles leads to expansion of specific human T cells," Journal of Virology, vol. 81, no. 3, pp. 1319-1326, 2007.

[68] S. C. Fausch, D. M. Da Silva, and W. M. Kast, "Differential uptake and cross-presentation of human papillomavirus virus-like particles by dendritic cells and Langerhans cells," Cancer Research, vol. 63, no. 13, pp. 3478-3482, 2003.

[69] C. Ruedl, T. Storni, F. Lechner, T. Bachi, and M. F. Bachmann, "Cross-presentation of virus-like particles by skin-derived CD8 ${ }^{-}$dendritic cells: a dispensable role for TAP," European Journal of Immunology, vol. 32, no. 3, pp. 818-825, 2002.

[70] X. Paliard, Y. Liu, R. Wagner, H. Wolf, J. Baenziger, and C. M. Walker, "Priming of strong, broad, and long-lived HIV type 1 p55(gag)-specific $\mathrm{CD}^{+}$cytotoxic T cells after administration of a virus-like particle vaccine in rhesus macaques," AIDS Research and Human Retroviruses, vol. 16, no. 3, pp. 273-282, 2000.

[71] P. Lacasse, J. Denis, R. Lapointe, D. Leclerc, and A. Lamarre, "Novel plant virus-based vaccine induces protective cytotoxic T-lymphocyte-mediated antiviral immunity through dendritic cell maturation," Journal of Virology, vol. 82, no. 2, pp. 785-794, 2008.

[72] V. G. Moron, P. Rueda, C. Sedlik, and C. Leclerc, "In vivo, dendritic cells can cross-present virus-like particles using an endosome-to-cytosol pathway," The Journal of Immunology, vol. 171, no. 5, pp. 2242-2250, 2003.

[73] W. Bohm, R. Schirmbeck, A. Elbe, et al., "Exogenous hepatitis B surface antigen particles processed by dendritic cells or macrophages prime murine MHC class I-restricted cytotoxic T lymphocytes in vivo," The Journal of Immunology, vol. 155, no. 7, pp. 3313-3321, 1995.

[74] P. Di Bonito, F. Grasso, S. Mochi, et al., "Anti-tumor CD8+ T cell immunity elicited by HIV-1-based virus-like particles incorporating HPV-16 E7 protein," Virology, vol. 395, no. 1, pp. 45-55, 2009.

[75] Y. Krishnamachari and A. K. Salem, "Innovative strategies for co-delivering antigens and CpG oligonucleotides," Advanced Drug Delivery Reviews, vol. 61, no. 3, pp. 205-217, 2009.

[76] M. Singh and D. T. O'Hagan, "Recent advances in vaccine adjuvants," Pharmaceutical Research, vol. 19, no. 6, pp. 715$728,2002$.

[77] X.-Q. Zhang, C. E. Dahle, N. K. Baman, N. Rich, G. J. Weiner, and A. K. Salem, "Potent antigen-specific immune responses stimulated by codelivery of CpG ODN and antigens in degradable microparticles," Journal of Immunotherapy, vol. 30, no. 5, pp. 469-478, 2007.

[78] M. E. Keegan and W. M. Saltzman, "Surface-modified biodegradable microspheres for DNA vaccine delivery," Methods in Molecular Medicine, vol. 127, pp. 107-113, 2006.
[79] Y. Waeckerle-Men and M. Groettrup, "PLGA microspheres for improved antigen delivery to dendritic cells as cellular vaccines," Advanced Drug Delivery Reviews, vol. 57, no. 3, pp. 475-482, 2005.

[80] F. Boisgerault, P. Rueda, C. M. Sun, S. Hervas-Stubbs, M. Rojas, and C. Leclerc, "Cross-priming of T cell responses by synthetic microspheres carrying a $\mathrm{CD}^{+} \mathrm{T}$ cell epitope requires an adjuvant signal," The Journal of Immunology, vol. 174, no. 6, pp. 3432-3439, 2005.

[81] S. R. M. Bennett, F. R. Carbone, F. Karamalis, J. F. A. P. Miller, and W. R. Heath, "Induction of a $\mathrm{CD}^{+}$cytotoxic T lymphocyte response by cross-priming requires cognate $\mathrm{CD} 4^{+}$ T cell help," Journal of Experimental Medicine, vol. 186, no. 1, pp. 65-70, 1997.

[82] S. R. M. Bennett, F. R. Carbone, F. Karamalis, R. A. Flavell, J. F. A. P. Miller, and W. R. Heath, "Help for cytotoxic-T-cell responses is mediated by CD4O signalling," Nature, vol. 393, no. 6684, pp. 478-480, 1998.

[83] Y. Waeckerle-Men, E. Scandella, E. Uetz-Von Allmen, et al., "Phenotype and functional analysis of human monocytederived dendritic cells loaded with biodegradable poly(lactideco-glycolide) microspheres for immunotherapy," Journal of Immunological Methods, vol. 287, no. 1-2, pp. 109-124, 2004.

[84] E. Schlosser, M. Mueller, S. Fischer, et al., "TLR ligands and antigen need to be coencapsulated into the same biodegradable microsphere for the generation of potent cytotoxic $\mathrm{T}$ lymphocyte responses," Vaccine, vol. 26, no. 13, pp. 16261637, 2008.

[85] L. Krishnan, K. Gurnani, C. J. Dicaire, et al., "Rapid clonal expansion and prolonged maintenance of memory $\mathrm{CD}^{+} \mathrm{T}$ cells of the effector $\left(\mathrm{CD} 44^{\text {high }} \mathrm{CD} 62 \mathrm{~L}^{\text {low }}\right)$ and central $\left(\mathrm{CD} 44^{\text {high }} \mathrm{CD} 62 \mathrm{~L}^{\text {low }}\right.$ ) phenotype by an archaeosome adjuvant independent of TLR2," The Journal of Immunology, vol. 178, no. 4, pp. 2396-2406, 2007.

[86] D. H. Busch, I. M. Pilip, S. Vijh, and E. G. Pamer, "Coordinate regulation of complex $\mathrm{T}$ cell populations responding to bacterial infection," Immunity, vol. 8, no. 3, pp. 353-362, 1998.

[87] A. Badiee, N. Davies, K. McDonald, et al., "Enhanced delivery of immunoliposomes to human dendritic cells by targeting the multilectin receptor DEC-205," Vaccine, vol. 25, no. 25, pp. 4757-4766, 2007.

[88] A. Badiee, M. R. Jaafari, and A. Khamesipour, "Leishmania major: immune response in BALB/c mice immunized with stress-inducible protein 1 encapsulated in liposomes," Experimental Parasitology, vol. 115, no. 2, pp. 127-134, 2007.

[89] L. Krishnan and G. D. Sprott, "Archaeosome adjuvants: immunological capabilities and mechanism(s) of action," Vaccine, vol. 26, no. 17, pp. 2043-2055, 2008.

[90] L. Krishnan, C. J. Dicaire, G. B. Patel, and G. D. Sprott, "Archaeosome vaccine adjuvants induce strong humoral, cellmediated, and memory responses: comparison to conventional liposomes and alum," Infection and Immunity, vol. 68, no. 1, pp. 54-63, 2000.

[91] K. Gurnani, J. Kennedy, S. Sad, G. D. Sprott, and L. Krishnan, "Phosphatidylserine receptor-mediated recognition of archaeosome adjuvant promotes endocytosis and MHC class I cross-presentation of the entrapped antigen by phagosometo-cytosol transport and classical processing," The Journal of Immunology, vol. 173, no. 1, pp. 566-578, 2004.

[92] L. Krishnan, S. Sad, G. B. Patel, and G. D. Sprott, "Archaeosomes induce enhanced cytotoxic T lymphocyte responses to entrapped soluble protein in the absence of interleukin 12 and 
protect against tumor challenge," Cancer Research, vol. 63, no. 10, pp. 2526-2534, 2003.

[93] L. Krishnan, S. Sad, G. B. Patel, and G. D. Sprott, "Archaeosomes induce long-term $\mathrm{CD}^{+}$cytotoxic $\mathrm{T}$ cell response to entrapped soluble protein by the exogenous cytosolic pathway, in the absence of $\mathrm{CD} 4^{+} \mathrm{T}$ cell help," The Journal of Immunology, vol. 165, no. 9, pp. 5177-5185, 2000. 


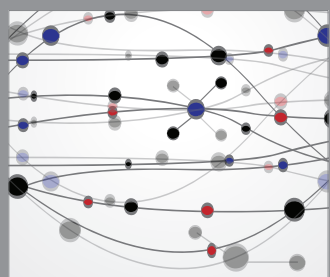

The Scientific World Journal
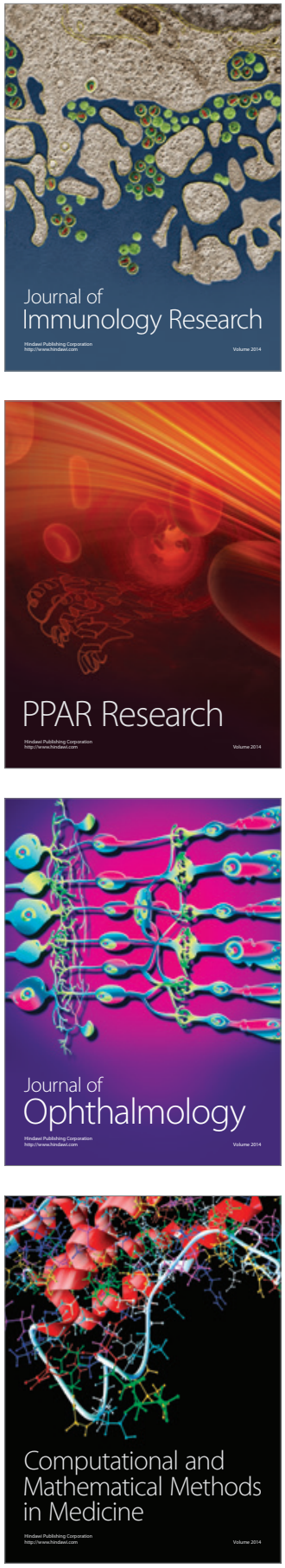

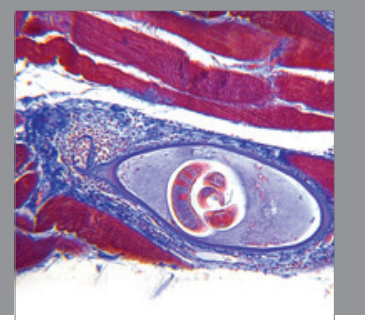

Gastroenterology

Research and Practice
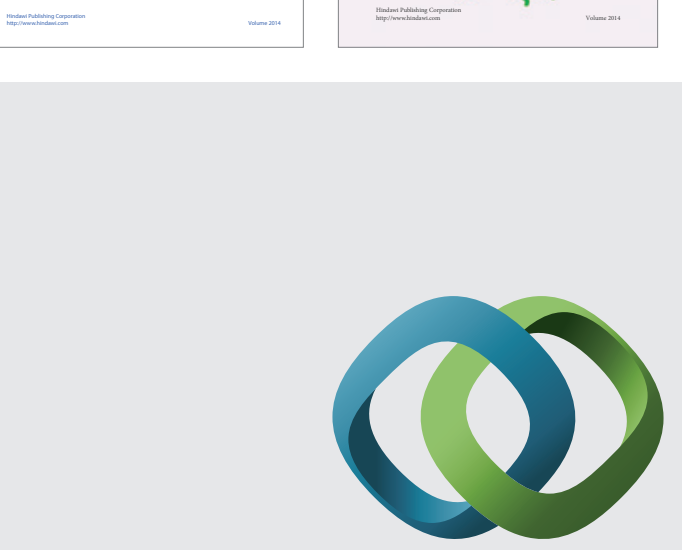

\section{Hindawi}

Submit your manuscripts at

http://www.hindawi.com
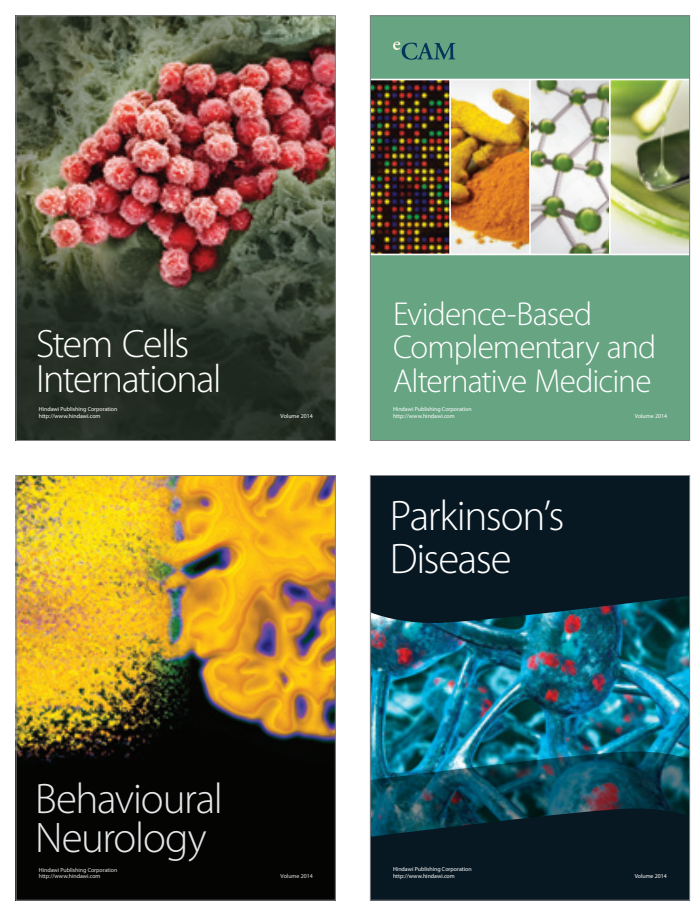

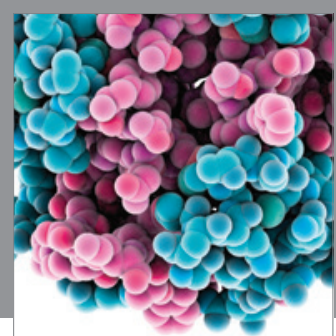

Journal of
Diabetes Research

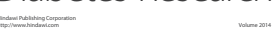

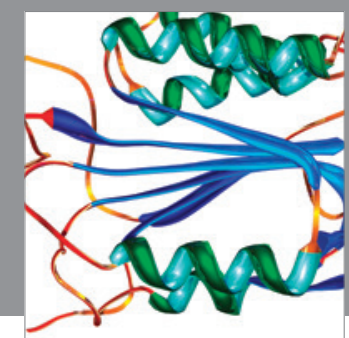

Disease Markers
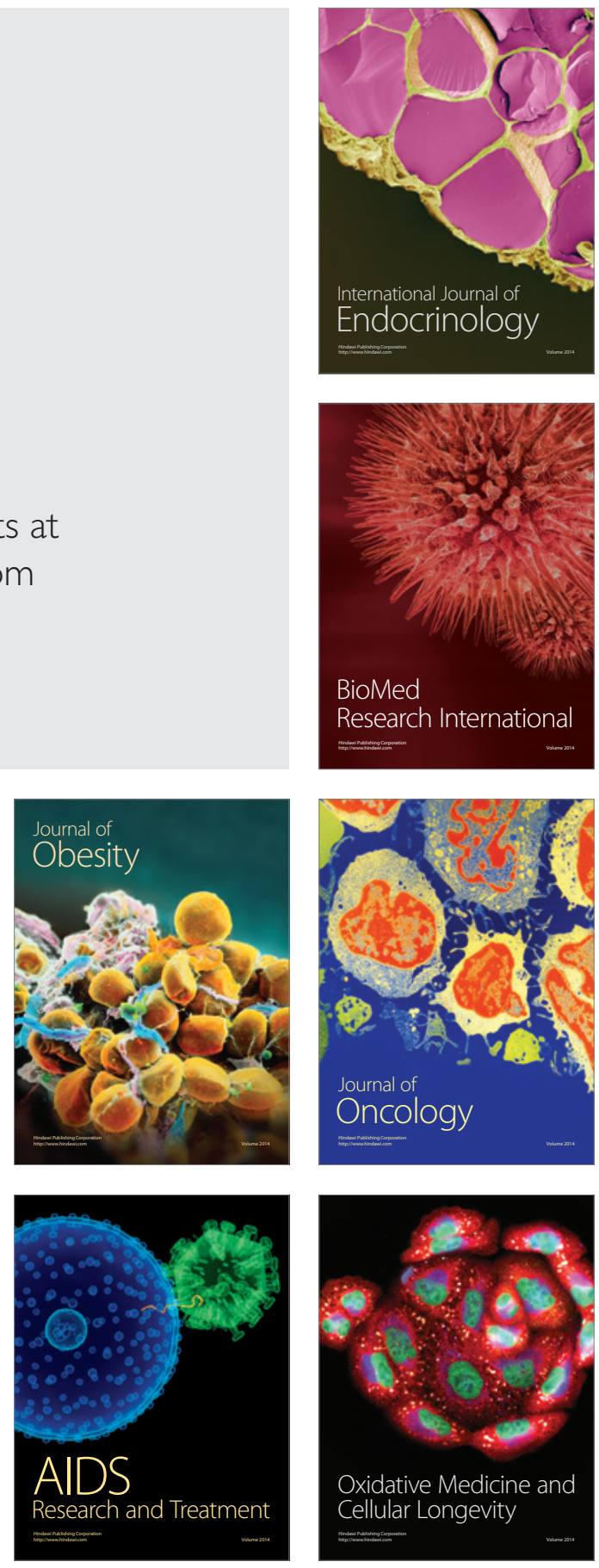